\title{
Single fibre EMG findings in polyneuropathies of different aetiology
}

\author{
BARBARA THIELE AND E. STÅLBERG \\ From the Departments of Clinical Neurophysiology, Klinikum Steglitz, Berlin, Germany, \\ and University Hospital, Uppsala, Sweden
}

SYNOPSIS Single fibre electromyography was carried out in patients with polyneuropathy due to uraemia, diabetes, and alcohol. In the two former groups the fibre density within the motor unit and the impulse transmission were mainly normal. In the latter group the fibre density was significantly increased as signs of reinnervation. Impulse transmission was impaired in a number of the action potential complexes, which is typical of active reinnervation. The results may indicate that the diabetic and uraemic polyneuropathies are characterized in the main by demyelination, whereas the alcoholic type is dominated by axonal lesion even at an early stage.

As is known from histological studies both in experimentally produced and in clinical polyneuropathies, the lesion in the peripheral nerve fibre can be restricted to the myelin sheath (demyelination) and can, in addition, affect the axon (Wallerian degeneration). In diphtheritic and allergic experimental polyneuropathy (Kaeser and Lambert, 1962; Cragg and Thomas, 1964; Hall, 1967; Weller and Nester, 1972), the lesion has been shown to be primarily restricted to the myelin sheath while with isoniazid (INH) axonal damage dominates (Schlaepfer and Hager, 1964).

In clinical polyneuropathies the differentiation between myelin and axonal lesions is of great importance, especially with regard to the prognosis for restitution of nerve function which is impaired with involvement only of myelin sheath.

Nerve biopsies are useful in this evaluation but have some limitations. They cannot be carried out in all patients, are restricted to a few nerves (distal in sensory nerves), and cannot be repeated in the same nerve.

With routine neurophysiological investigations, it may also be difficult to differentiate between the two types of polyneuropathy. In both types the impulse propagation velocity is

(Accepted 21 April 1975.) reduced in the peripheral nerves, although more pronounced with myelin lesions (Kaeser and Lambert, 1962). In both forms of polyneuropathy the concentric needle EMG shows a reduced interference pattern and changes in potential form and duration. Those cases with more pronounced axonal lesions show fibrillation potentials and with reinnervation an increase in amplitude and duration of the motor unit action potentials.

The bases of the electromyographic differentiation between axonal and myelin lesions are thus the signs of reinnervation in the former type. The single fibre electromyographic technique (SFEMG) has in earlier studies been shown to be a sensitive method for detecting signs of reinnervation (Stålberg and Ekstedt, 1973; Hakelius and Stålberg, 1974). The aim of the present investigation was to evaluate whether SFEMG offered possibilities of discriminating between axonal and myelin lesions in slight and moderate polyneuropathies.

\section{METHODS}

Fifty-four patients were investigated (Table). Most of the patients had been evaluated clinically by a neurologist and the diagnosis of polyneuropathy was based on symptoms such as paraesthesia, reduced 881 
TABLE

FINDINGS IN THREE GROUPS OF PATIENTS AND IN NORMAL SUBJECTS

\begin{tabular}{|c|c|c|c|c|c|c|c|c|c|}
\hline \multirow[t]{2}{*}{ Aetiology } & \multirow[t]{2}{*}{$n$} & \multicolumn{2}{|c|}{ Age $(y r)$} & \multirow[t]{2}{*}{$F D$} & \multirow{2}{*}{$\begin{array}{c}\text { Difference compared } \\
\text { with normal of } \\
\text { corresponding age } \\
P \text {-value }\end{array}$} & \multicolumn{4}{|c|}{ Motor nerve conduction velocity $(\mathrm{m} / \mathrm{s})$} \\
\hline & & Range & Mean $S D$ & & & Median & Ulnar & Peroneal & $\begin{array}{c}\text { Tibialis } \\
\text { posterior }\end{array}$ \\
\hline $\begin{array}{l}\text { Uraemia } \\
\text { Diabetes } \\
\text { Alcohol } \\
\text { Normal }\end{array}$ & $\begin{array}{r}17 \\
19 \\
18 \\
100\end{array}$ & $\begin{array}{l}23-71 \\
22-80 \\
24-70 \\
20-69\end{array}$ & $\begin{array}{l}53.7 \pm 11.2 \\
58.9 \pm 12.3 \\
44.0 \pm 11.7\end{array}$ & $\begin{array}{l}1.58 \pm 0.26 \\
1.52 \pm 0.19 \\
2.03 \pm 0.72 \\
1.48 \pm 0.15\end{array}$ & $\begin{array}{l}>0.1 \\
>0.3 \\
<0.005\end{array}$ & $\begin{array}{l}49.1 \pm 6.0 \\
48.9 \pm 3.0 \\
52.1 \pm 6.5\end{array}$ & $\begin{array}{l}52.5 \pm 7.2 \\
49.1 \pm 5.6 \\
52.5 \pm 6.2\end{array}$ & $\begin{array}{l}37.6 \pm 4.6 \\
35.3 \pm 3.5 \\
40.0 \pm 5.5\end{array}$ & $\begin{array}{c}36.8 \pm 4.1 \\
37.5 \pm 3.3 \\
40.2 \pm 4.4\end{array}$ \\
\hline
\end{tabular}

ances of tactile and vibration sensibility, and muscular atrophy.

Conventional EMG with concentric needle electrode was carried out in half of the patients in the tibialis anterior muscle and somewhat less often in the first dorsal interosseous muscle.

In all patients, motor and sensory conduction velocity was measured in three or more peripheral nerves. The motor and antidromic sensory conduction velocities were measured in the conventional way with surface electrodes. Only patients with reduced conduction velocities in at least two motor nerves were included in the study. A lower limit of normal conduction velocity was (Thomas et al., 1959): ulnar and median nerve: motor conduction velocity $50 \mathrm{~m} / \mathrm{s}$; sensory conduction velocity 55 $\mathrm{m} / \mathrm{s}$; peroneal and tibial nerve: motor conduction velocity $40 \mathrm{~m} / \mathrm{s}$. The action potentials recorded with the SFEMG-electrode ( $25 \mu \mathrm{m}$ recording surface) were amplified on a Disa equipment and displayed on a 565 Tektronix oscilloscope. They were stored on magnetic tape (AKAI X300).

Analysis of data was made either on-line during recording on the oscilloscope screen (Tektronix D15 storage) or off-line by a Linc 8 computer. The single fibre EMG investigation was performed in the extensor digitorum communis muscle (EDC) despite the fact that signs of polyneuropathy usually are more pronounced in the legs than in the arms. This muscle was chosen as it is easy to activate even for weak and otherwise disabled patients and was found to show only slight age dependent changes before the age of 60 years. (Stålberg and Thiele, 1975).

SINGLE FIBRE EMG PARAMETERS Fibre density The fibre density is measured as the mean number of muscle fibres belonging to a single motor unit within the uptake radius of the electrode $(270 \mu \mathrm{m})$. On random insertions activity from one fibre is recorded in $60-70 \%$, from two fibres in about $30 \%$, and more seldom from three or more fibres. This can be expressed as a mean fibre density of $1.25-1.7$ fibres/ electrode uptake area in normal subjects. This value is rather constant until the age of 60 years, after which it gradually increases. The method and normal values are presented by Stålberg and Thiele (1975).

Maximal action potential duration Action potentials from two or more muscle fibres from the same motor unit are recorded and the time between the zero intersection point of the first and last component is measured.

Jitter When recording is made from two or more muscle fibres from the same motor unit, a variability is seen in the interpotential interval between the spike components at consecutive discharges, the jitter. This is an expression of the variability in impulse transmission over the motor endplates but also along the nerve fibres distally to the last branching point and along the muscle fibres under investigation. The normal value is $5-50 \mu \mathrm{s}$, expressed as the mean value of consecutive difference of the interpotential intervals (Stålberg et al., 1971). With disturbed transmission in the mentioned structures the jitter is increased and in more pronounced impairment impulse blocking appears (Stålberg and Ekstedt, 1973).

\section{RESULTS}

In all three groups of patients the duration of symptoms exceeded six months. Clinically, the symptoms were classified as mild or moderate. Most affected were some of the patients with alcoholic polyneuropathy. In the diabetic and uraemic group sensory symptoms dominated, in the alcoholic group weakness and muscular atrophies. Motor nerve conduction velocities were, on average, less reduced in the alcoholic group.

Concentric needle EMG showed in the 

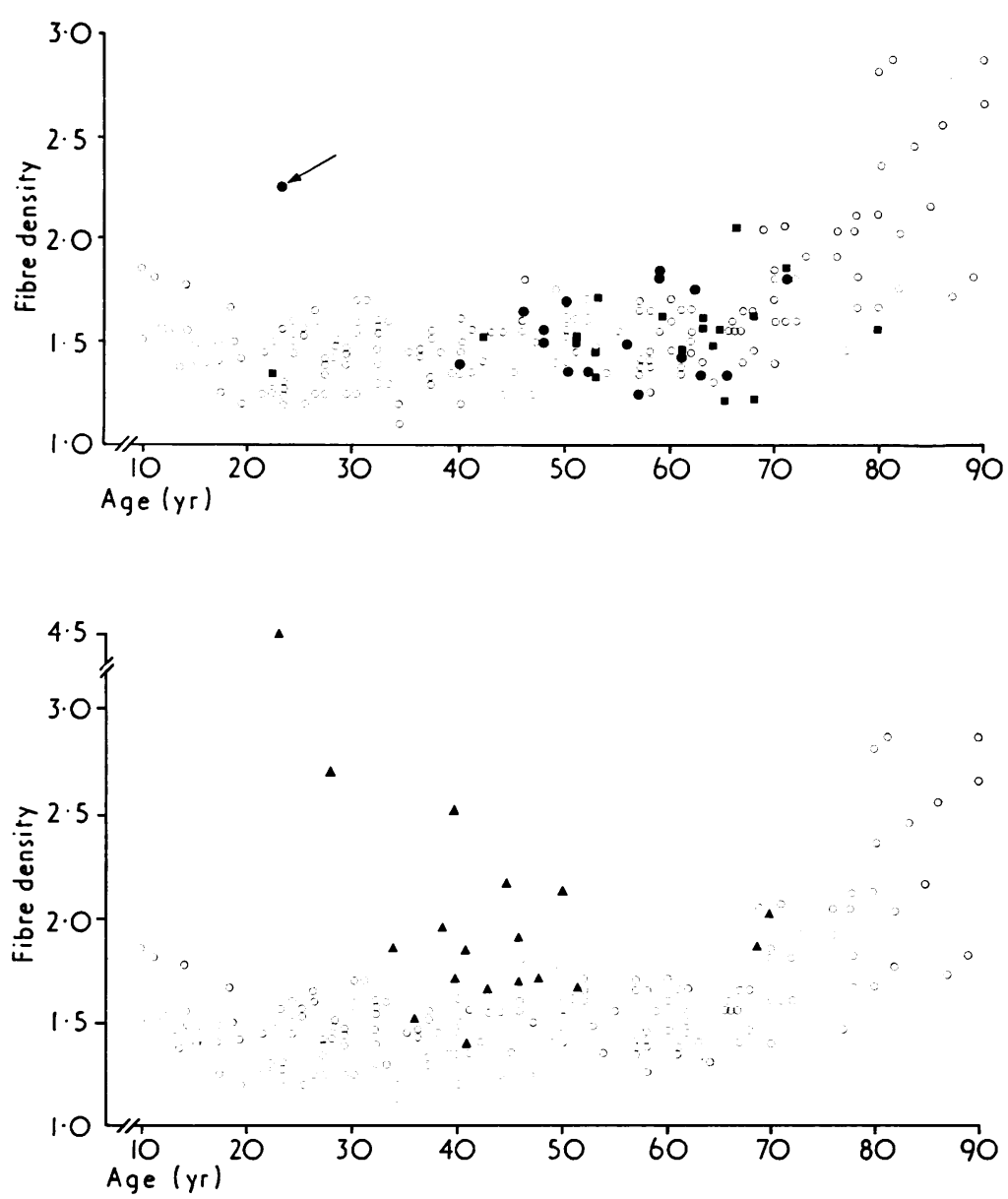

FIG. 1 Comparison of fibre density values measured in patients with uraemic $(\mathbf{O})$ and diabetic polyneuropathy $(\square)$ and normal $(O)$. Age on the abscissa, fibre density on the ordinate. One patient with uraemic polyneuropathy $(\leftarrow)$ is outside the normal range. He had been on nitrofurantoin treatment.

FIG. 2 Comparison of fibre density values measured in patients with alcoholic polyneuropathy $(\mathbf{\Delta})$ and in normal subjects $(\bigcirc)$. Note markedly increased fibre density, especially in the younger alcoholics. uraemic and diabetic group: scanty fibrillation potentials, mainly normal motor unit potentials, and moderately reduced interference pattern. In the alcoholic group: frequent fibrillation potentials, increased number of polyphasic long duration motor unit potentials, and moderately or occasionally markedly reduced interference pattern.

URAEMIA, DIABETES Despite a pronounced reduction in conduction velocity in peripheral nerves in some patients with uraemic and diabetic polyneuropathy the SFEMG parameters of fibre density and potential duration were surprisingly normal (Figs 1 and 3). The mean value of fibre density calculated for the whole material was $1.58 \pm 0.26$ for uraemia and
$1.52 \pm 0.19$ for diabetes, which does not differ significantly from the normal value for the age group 20-69 years, $1.48 \pm 0.15(\mathrm{P}>0.1$ and $>0.3$ respectively). One diabetic patient, aged 80 years, had a fibre density of 1.55 . If the next decade 70-79 years, of the normal subjects had been included to correspond with this single patient, the normal values were $1.53 \pm 0.16$ and the difference between the patient group and the normal subjects would have been even smaller. One patient in the uraemic group and none in the diabetic group had fibre density values exceeding 3SD from the normal mean value for the corresponding age decade. The fibre density did not differ significantly between the two groups $(P>0.4)$. A slightly increased incidence of potentials with duration beyond 
$3 \mathrm{~ms}$ (normal $1.2 \%$ ) could be seen in patients with uraemic polyneuropathy $(4.8 \%)$ and to a lesser degree in diabetic polyneuropathy $(3.8 \%)$ (Fig. 3). A jitter exceeding $50 \mu$ s but without blocking was seen in uraemic polyneuropathy in about $2 \%$ of the recorded multiple motor unit potentials and in diabetic about $3 \%$ as com-

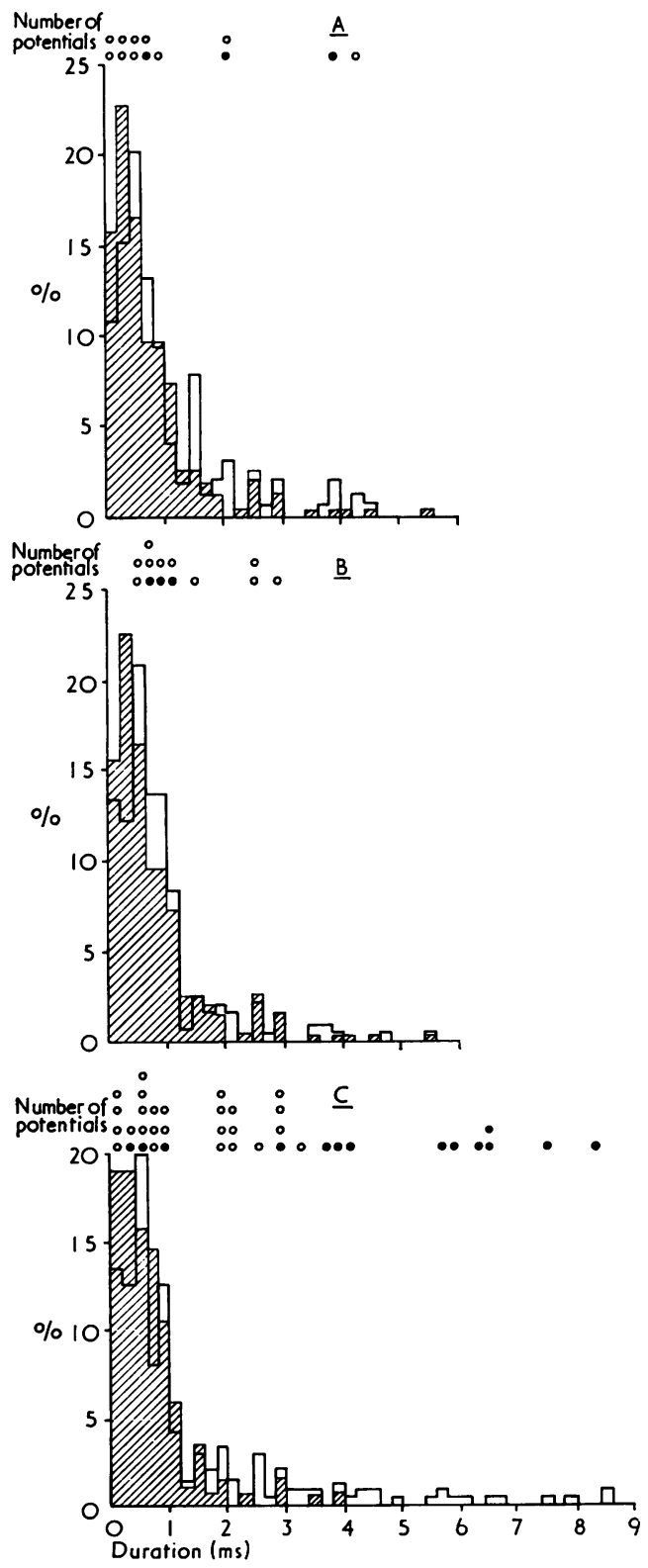

pared with $0.6 \%$ in the corresponding age groups of normal subjects. The incidence of impulse blockings was within normal limits (about $1 \%$ of the recorded multiple potentials) for both groups of patients. One young patient with uraemia was outstanding in the material (Fig. 1) concerning fibre density, jitter, and blocking. He was the only case earlier treated with nitrofurantoin.

ALCOHOL In alcoholic polyneuropathy, the fibre density values were above or in the upper range of the normal material and exceeding 3SD from the normal mean values in seven of the 18 patients (Fig. 2). The fibre density value for the patients was $2.03 \pm 0.72$ as compared with $1.48 \pm 0.15$ in normal subjects $(P<0.005)$. Single potentials were recorded in about $40 \%$ of random insertions but multiple action potentials, with up to nine components, were seen more frequently than in normal subjects and than in the uraemic and diabetic polyneuropathies. The values for the two youngest patients (24 and 28 years of age) were 4.6 and? 2.65 respectively, which is far outside the normale range for this age (1.38). Furthermore, action $\frac{\mathbb{}}{0}$ potential complexes of prolonged duration were often recorded, with $10.4 \%$ of the multiple potentials exceeding $3 \mathrm{~ms}$ (normal $1.2 \%$ ) (Fig. 3) There was an increase in jitter and blockings. The incidence of jitter above $50 \mu \mathrm{s}$ was about $6 \%$ as compared with $0.3 \%$ in normal subjects and blockings were seen in about $3 \%$ compared with $0 \%$ in normal subjects of the corresponding age. Blockings were seen especially in the late components of the prolonged multiple potential recordings.

FIG. 3 Duration of the recorded multiple potentials. A: uraemic polyneuropathy, B: diabetic polyneuropathy, C: alcoholic polyneuropathy. Corresponding age groups of normal subjects are shaded in the histogram. Abscissa: duration; ordinate: percentage of incidence. Prolonged action potential duration was seen especially in alcoholic polyneuropathy and to a lesser degree in uraemic-diabetic polyneuropathy. Individual potentials with increased jitter $(O)$ and with blockings (O) are indicated for the respective duration in the upper part of each histogram. 


\section{DISCUSSION}

In uraemic and diabetic polyneuropathy the fibre density and jitter did not differ significantly from normal and no significant difference was found between these two groups of patients. The number of potentials with a duration exceeding $3 \mathrm{~ms}$ was slightly above normal in both groups. Patients with alcoholic polyneuropathy, however, showed significantly increased fibre density, along with jitter and blocking and potentials with prolonged duration. The findings in this group are interpreted as signs of reinnervation after preceding denervation.

The difference between the two groups of polyneuropathy may be due either to patient selection factors or to major difference in the pathophysiology. It might be suspected that patients with symptoms of alcoholic polyneuropathy come to the doctor after a longer delay than those with diabetes or uraemia who are usually under continuous medical supervision. It was not possible to obtain sufficiently accurate information about the duration of symptoms to allow a detailed comparison between the different groups of patients in the material presented but the duration of symptoms of polyneuropathy always exceeded six months. The patients with alcoholic polyneuropathy were usually more affected clinically, but the nerve conduction velocity was less reduced than in the other two groups.

A more plausible explanation for the difference seems to be a different pathophysiological mechanism in diabetic and uraemic polyneuropathies than in the alcoholic form.

DIABETIC AND URAEMIC POLYNEUROPATHY Could the missing signs of reinnervation be due to the fact that the nerve damage is present only in the sensory nerves? This is not likely as the motor conduction velocity was reduced in at least two peripheral motor nerves in each patient. Could axonal damage have taken place without succeeding signs of reinnervation due to loss of regenerative power in the intramuscular nerve twigs? This has been shown in muscle biopsies in juvenile diabetes by Reske-Nielssen et al. (1970). The findings in the oldest diabetic patient $(80$ years of age), where the fibre density value 1.55 is lower than found in the normal material, might indicate a reduced regenerative capacity not compensating to the usual extent for the general age-dependent degeneration and not for the denervation possibly caused by the polyneuropathy. Another explanation for the missing signs of reinnervation would be that no denervation had taken place - that is, no axonal damage. The scantiness of fibrillation potentials in the concentric needle EMG supports this assumption.

For diabetic polyneuropathy this suggestion is supported by other investigations where it has been shown that the most common type of lesion in nerve biopsies was a demyelination (Dolman, 1963; Thomas and Lascelles, 1965; Chopra et al., 1968; Bischoff, 1970; Noel, 1973). Axonal lesions have been described only occasionally, more often in severe and chronic cases (Noel, 1973). An affection of spinal ganglia, spinal nerve roots, and ventral horn cells in diabetes is also described (Dolman, 1963; Gibbels and Schliep, 1970). The most likely cause for missing signs of reinnervation in our generally early and slightly or moderately involved cases seems to be that the polyneuropathy is initially mainly of demyelinating type.

In uraemic polyneuropathy the nerve biopsy findings presented in the literature are contradictory. While Jennekens et al. (1961) and Dyck et al. (1971) described predominantly axonal lesions, others found the lesion to be primarily a demyelination with a small amount of secondary axonal involvement (Asbury et al., 1963; Dayan et al., 1970; Dinn and Crane, 1970). The differing findings may be due to the dynamics of the process. A long-standing demyelination may cause a damage of the axon because of its increased sensitivity to mechanical and toxic influences. Our material showed very scanty signs of reinnervation. The exception was one case, clinically not the most pronounced, but with earlier nitrofurantoin therapy. From experiments on rats it is known that this drug leads to axonal lesions (Klinghardt, 1967). Whether the lack of reinnervation in uraemic polyneuropathy is due to a restricted demyelination, as in diabetes, or to insufficient regeneration cannot be settled definitely. The absence of fibrillation potentials and the slow nerve conduction velocity within the same range as found in diabetes are indicative of a demyelination. The prolonged duration of the multiple motor unit action poten- 
tials in the presented study can be explained by the slow conduction in the terminal nerve tree. If a pronounced axonal lesion exists in these cases without reinnervation, a marked atrophy is to be expected, which was not seen in our cases. Thus, as in diabetic polyneuropathy, the lesion seems mainly to be a demyelination.

ALCOHOLIC POLYNEUROPATHY Increased fibre density compared with normal was noted in the alcoholic polyneuropathy. This is a SFEMG sign of reinnervation correlated with the fibre grouping seen in the reinnervated muscle after partial nerve lesion (Karpati and Engel, 1968). Also, the conventional EMG findings indicated a relatively pronounced axonal damage with fibrillation potentials, signs of reinnervation, and drop out of motor units. In alcoholic polyneuropathy, the increased potential duration along with increased jitter and increased incidence of potential blocking can be assumed to be due to impaired impulse conduction in newly formed reinnervation sprouts (Stålberg and Thiele, 1972). Impulse blocking indicates active reinnervation during the previous three to six months (Hakelius and Stålberg, 1974) provided that the functioning nerve fibres have a reinnervation capacity similar to those which reinnervate a traumatically denervated muscle fibre. The relatively low number of action potentials with increased jitter and blocking in alcoholic polyneuropathy indicates a relatively slowly progressive process.

Axonal involvement is confirmed by most of the nerve biopsy findings (Gilliatt, 1966; Dyck et al., 1968; Walsh and McLeod, 1970). Bischoff $(1970,1971)$ described, however, an equal incidence of myelin or axonal lesions in nerve biopsies in patients with alcoholic polyneuropathy and assumed different pathophysiological mechanisms - that is, primary affection of the myelin sheath or of the axon. The demyelinating type may be due to impaired liver function (Knill-Jones et al., 1972) rather than to a direct toxic effect of the alcohol.

In our material with alcoholic polyneuropathy, all but two patients showed the SFEMG signs of reinnervation. The younger the patients the more pronounced were the signs of reinnervation. The two patients older than 70 years of age had fibre density values at the upper limit of normal indicating a possible loss of regenerative power in higher ages.

Muscular atrophies, although usually mild, were common in alcoholic polyneuropathy in our material, while the patients with diabetic and uraemic polyneuropathy complained mostly of sensory symptoms. For an equal degree of demyelination in motor and sensory nerves, symptoms are expected to be more pronounced for the sensory system. The demyelinated nerve fibres show an impaired but not totally blocked impulse transmission, especially at higher impulse rates (Lehmann and Tackmann, 1970). This can be compensated for in the motor system by increasing the innervation rate and by recruitment but not in the sensory system, where it leads to a loss of tactile information.

With the SFEMG method, the diabetic and uraemic polyneuropathies on one hand, and alcoholic polyneuropathy on the other, show differences, particularly as far as fibre density is concerned, which most likely reflect different pathological processes. It may be possible to differentiate polyneuropathies with mainly axonal damage from those with mainly myelin lesion. The parameters that are described might be of value in the investigations of other types of polyneuropathies - for example, the toxic forms found in occupational medicine.

The investigation was supported by the Swedish Medical Research Council (Grant No. 04X-135). Kerstin Mellqvist is acknowledged for her technical assistance. Gösta Lovén constructed the SFEMG electrodes.

\section{REFERENCES}

Asbury, A. K., Victor, M., and Adams, R. D. (1963) Uremic polyneuropathy. Archives of Neurology (Chic.), 8, 413-428.

Bischoff, A. (1970). Erkrankungen des peripheren Nervensystems bei Diabetes mellitus und Alkoholabusus. Arztliche Fortbildung, 18, 272-276.

Bischoff, A. (1971). Alkoholische Polyneuropathieklinische ultrastrukturelle und pathogenetische Aspekte. Deutsche Medizinische Wochenschrift, 96, 317-322.

Chopra, J. S., Hurwitz, L. J., and Montgomery, D. A. D. (1968). The pathogenesis of sural nerve changes in diabetes mellitus. Brain, 92, 391-418.

Cragg, B. G., and Thomas, P. K. (1964). Changes in nerve conduction in experimental allergic neuritis. Journal of Neurology, Neurosurgery, and Psychiatry, 27, 106-115.

Dayan, A. D., Gardner-Thorpe, C., Down, P. F., and Gleadle, J. (1970). Peripheral neuropathy in uremia. Neurology (Minneap.), 20, 649-658.

Dinn, J. J., and Crane, D. L. (1970). Schwann cell dysfunction in uraemia. Journal of Neurology, Neurosurgery, and Psychiatry, 33, 605-608. 
Dolman, C. L. (1963). The morbid anatomy of diabetic neuropathy. Neurology (Minneap.), 13, 135-142.

Dyck, P. J., Gutrecht, J. A., Bastron, J. A., Karnes, W. E., and Dale, A. J. D. (1968). Histologic and teased fibre measurements of sural nerve in disorders of lower motor and primary sensory neurones. Proceedings of the Staff Meetings of the Mayo Clinic, 43, 81.

Dyck, P. J., Johnson, W. J., Lambert, E. H., and O'Brien, P. C. (1971). Segmental demyelination secondary to axonal degeneration in uremic neuropathy. Proceedings of the Staff Meetings of the Mayo Clinic, 45, 400-431.

Gibbels, E., and Schliep, G. (1970). Diabetische Polyneuropathie-Probleme der Diagnostik und Nosologie. Fortschritte der Neurologie, Psychiatrie und ihrer Grenzgebiete, 38, 369-436.

Gilliatt, R. W. (1966). Nerve conduction in human and experimental neuropathies. Proceedings of the Royal Society of Medicine, 59, 989-993.

Hakelius, L., and Stålberg, E. (1974). Electromyographical studies of free autogenous muscle transplants in man. Scandinavian Journal of Plastic and Reconstructive Surgery, 8, 211-219.

Hall, J. H. (1967). Studies on demyelinated peripheral nerves in guinea-pig with experimental allergic neuritis, Part II, Electrophysiological observations. Brain, 90, 313-332.

Jennekens, F. G., van der Most van Spijk, D., Dorhout, and Mees, E. D. (1961). Nerve fibre degeneration in uremic polyneuropathy. Proceedings of the European Dialysis and Transplant Association, 4, 191-197.

Kaeser, H. E., and Lambert, E. H. (1962). Nerve function studies in experimental polyneuritis. Electroencephalography and Clinical Neurophysiology, Suppl. 22, 29-35.

Karpati, G., and Engel, W. K. (1968). Type grouping in skeletal muscles after experimental reinnervation. Neurology (Minneap.), 18, 447-455.

Klinghardt, G. W. (1967). Schädigungen des Nervensystems bei der Ratte durch Nitrofurane. Acta Neuropathology, 9, 18-33.

Knill-Jones, R. P., Goodwill, C. J., Dayan, A. D., and Williams, R. (1972). Peripheral neuropathy in chronic liver disease: clinical electrodiagnostic and nerve biopsy findings. Journal of Neurology, Neurosurgery, and Psychiatry, 35, 2230.

Lehmann, H. J., and Tackmann, W. (1970). Die Übermitt- lung frequenter Impulsserien in demyelinisierten und in degenerierten Nervenfasern. Archiv für Psychiatrie und Nervenkrankheiten, 213, 215-227.

Noel, P. (1973). Diabetic neuropathy. In New Developments in Electromyography and Clinical Neurophysiology, vol. 2, pp. 318-332. Karger: Basel.

Reske-Nielsen, E., Lundbaek, K., Gregersen, G., and Harmsen, A. (1970). Pathological changes in the central and peripheral nervous system of young long term diabetics. Diabetologica, 6, 98-103.

Schlaepfer, W. W., and Hager, H. (1964). Ultrastructural studies of INH induced neuropathy in rats: 1. Early axonal changes. American Journal of Pathology, 45, 209219.

Stålberg, E., and Ekstedt, J. (1973). Single fibre EMG and microphysiology of the motor unit in normal and diseased human muscle. In New Developments in Electromyography and Clinical Neurophysiology, vol. 1, pp. 113-129. Karger: Basel.

Stålberg, E., Ekstedt, J., and Broman, A. (1971). The electromyographic filter in normal human muscles. Electroencephalography and Clinical Neurophysiology, 31, 429438.

Stålberg, E., and Thiele, B. (1975). Motor unit fibre density in the extensor digitorum communis muscle. Journal of Neurology, Neurosurgery, and Psychiatry, 38, 874-880.

Stålberg, E., and Thiele, B. (1972). Transmission block in terminal nerve twigs. A single fibre electromyographic finding in man. Journal of Neurology, Neurosurgery, and Psychiatry, 35, 52-59.

Thomas, P. K., Sears, T. A., and Gilliatt, R. W. (1959). The range of conduction velocity in normal motor nerve fibres to the small muscles of the hand and foot. Journal of Neurology, Neurosurgery, and Psychiatry, 22, 175-181.

Thomas, P. K., and Lascelles, R. G. (1965). Schwann-cell abnormalities in diabetic neuropathy. Lancet, 1, 1355-1357.

Walsh, J. C., and McLeod, J. G. (1970). Alcoholic neuropathy. An electrophysiological and histological study. Journal of the Neurological Sciences, 10, 457-469.

Weller, R. O., and Nester, B. (1972). Early changes at the node of Ranvier in segmental demyelinisation. Histochemical and electron microscopic observations. Brain, 95, 665-674. 